\title{
Red blood cell distribution width as a predictor of survival in nasal-type, extranodal natural killer/T-cell lymphoma
}

\author{
Huaichao Luo ${ }^{1, *}$, Xiaoying Quan ${ }^{2, *}$, Xiao-Yu Song ${ }^{1, *}$, Li Zhang ${ }^{1, *}$, Yilin Yin ${ }^{3, *}$, Qiao \\ $\mathrm{He}^{1}$, Shaolei Cai ${ }^{4}$, Shi Li ${ }^{1}$, Jian Zeng ${ }^{1}$, Qing Zhang ${ }^{1}$, Yu Gao ${ }^{1}$ and Sisi Yu ${ }^{2}$ \\ ${ }^{1}$ Department of Clinical Laboratory, Sichuan Cancer Hospital \& Institute, Sichuan Cancer Center, School of Medicine, \\ University of Electronic Science and Technology of China, Chengdu, Sichuan, China \\ ${ }^{2}$ Department of Medical Oncology, Sichuan Cancer Hospital \& Institute, Sichuan Cancer Center, School of Medicine, University \\ of Electronic Science and Technology of China, Chengdu, Sichuan, China \\ ${ }^{3}$ Department of Biology, The Northeastern University, Huntington Ave, Boston, MA, USA \\ ${ }^{4}$ Radiotherapy Center, Sichuan Cancer Hospital \& Institute, Sichuan Cancer Center, School of Medicine, University of Electronic \\ Science and Technology of China, Chengdu, Sichuan, China \\ *These authors have contributed equally to this work \\ Correspondence to: Sisi Yu, email: sisi-yu@hotmail.com \\ Huaichao Luo, email: lvo1987cc@163.com
}

Keywords: red blood cell distribution width (RDW), extranodal natural killer (NK)/T-cell lymphoma, predictor, survival, radiotherapybased treatment

Received: June 12, $2017 \quad$ Accepted: August 26, $2017 \quad$ Published: September 30, 2017

Copyright: Luo et al. This is an open-access article distributed under the terms of the Creative Commons Attribution License 3.0 (CC BY 3.0), which permits unrestricted use, distribution, and reproduction in any medium, provided the original author and source are credited.

\section{ABSTRACT}

We retrospectively enrolled 191 nasal-type, extranodal natural killer/T-cell lymphoma (ENKTL) patients newly diagnosed from 2008 to 2016 at the Sichuan Cancer Hospital, in order to evaluate the relationship between disease outcomes, demographic and clinical factors, and red blood cell distribution width (RDW). C-index, fisher's exact test, univariate analysis, and cox regression analysis were applied. The median age of patients was 44 years and $134(70 \%)$ were men. The cutoff of RDW was $46.2 \mathrm{fL}$ determined by Cutoff Finder. Patients with RDW $\leq \mathbf{4 6 . 2} \mathrm{fL}$ had significantly better progression-free survival (PFS) (3-year PFS, 80.4\% vs. $63.1 \%$; $P=0.01$ ) and overall survival (OS) (3-year OS, $83.2 \%$ vs. $65.5 \% ; P=0.004$ ) than those with RDW>46.2 fL. Multivariate analysis demonstrated that elevated RDW is an independent adverse predictor of OS $(P=0.021, H R=2.04)$. RDW is an independent predictor of survival outcomes in ENKTL, which we found to be superior to both the prognostic index of natural killer lymphoma (PINK) and the Korean Prognostic Index (KPI) in discriminating patients with different outcomes in low-risk and highrisk groups (all $P<0.05$ ). The new models combining RDW with the International Prognostic Index (IPI), KPI, and PINK showed more powerful prognostic value than corresponding original models. RDW represents an easily available and inexpensive marker for risk stratification in patients with ENKTL treated with radiotherapy-based treatment. Further prospective studies are warranted to confirm the prognostic value of RDW in ENKTL.

\section{INTRODUCTION}

Nasal-type, extranodal natural killer/T-cell lymphoma (ENKTL) is a highly aggressive and heterogeneous non-Hodgkin lymphoma (NHL). It is most common in East Asia and Latin America (upwards of 10\% of all NHLs), but rare in North America and Europe (less than $1 \%$ of all NHL) [1]. ENKTL accounts for $5-10 \%$ of 
all malignant lymphomas in China [2]. Depending on the primary site of the cancer, ENKTL is divided into upper aerodigestive tract NK/T-cell lymphoma (UNKTL) and extra-upper aerodigestive tract NK/T-cell lymphoma (EUNKTL). UNKTL typically causes the vast majority of cases of "lethal midline granuloma", a syndrome which involves the paranasal sinuses, orbits, and lymph nodes, and even develops outside of the nasopharynx [1].

The red blood cell distribution width (RDW), which is traditionally known as anisocytosis, reflects the size heterogeneity of erythrocyte volume. RDW has been used to discriminate between different types of anemia [3]; however, emerging evidence suggests that anisocytosis is involved in a variety of human disorders such as cardiovascular disease, venous thromboembolism, diabetes, community-acquired pneumonia, as well as other acute or chronic conditions [3]. The role of RDW in patients with various cancers was investigated by several studies [4], and RDW has proven to be an independent prognosis factor in breast cancer [5], esophageal squamous cell carcinoma [6], lung cancer [7], and diffuse large B-cell lymphoma (DLBCL) [8]. However to the best of our knowledge the prognostic value of RDW in T-cell NHL has never been investigated. Therefore, we performed this study to evaluate the prognostic significance of RDW in patients with ENKL.

\section{RESULTS}

\section{Patient characteristics}

The main baseline characteristics of the 191 patients studied are listed in Table 1. The median age was 44 years (range $=15-86$ years). Most of enrolled patients were male (134, 70.2\%). There was an Ann-Arbor (AA) staging I/II predominance. In all, 184 patients $(96.3 \%)$ were classified into AA I/II and 7 cases (3.7\%) into stage III/IV. The mean RDW was 45.7 (range $=36.3-65.0$ ) (data not shown).

The optimal cut-off value of RDW for OS and PFS was determined by Cutoff Finder via the method of ROC curve (Euclidean distance). These two outcomes have the same cut-off value of $46.2 \mathrm{fL}$. For OS and PFS, the areas under the curve (AUC) were 0.60 and 0.56 , sensitivities were 61.1 and 56.9, and specificities were 62.8 and 61.7 , respectively (Figure 1). In all, 107 (56.0\%) cases had low RDW ( $\leq 46.2 \mathrm{fL}$ ) and were classified into the low-risk group, whereas 84 (44\%) patients had high RDW (>46.2 fL) and were classified into the high-risk group. The highrisk group tended to have a higher death ratio than that of the low-risk group (38.8\% and $19.4 \%$, respectively). Regional lymphadenopathies were more frequent in patients with low RDW than in other patients. Patients in the high-risk group tended to have ECOG PS $\geq 2$, and IPI scores 2-5. Other characteristics showed no significant differences across high- or low-risk groups with different RDW levels (Table 1).

\section{Treatment modalities and response}

Treatment modalities and responses are listed in Table 2. Of 191 patients, 40 (20.9\%) received radiotherapy alone while $151(79.1 \%)$ had chemotherapy combined with radiation. Of those 151 patients, 55 (28.7\%) cases received chemotherapy followed by radiotherapy, 22 (11.5\%) had concurrent chemoradiotherapy, 14 (7.3\%) had concurrent chemoradiotherapy followed by chemotherapy, 45 (23.6\%) had chemotherapy before and after radiotherapy, and 15 (7.8\%) had radiotherapy followed by chemotherapy. The chemotherapy regimens included VDLP (4 patients), LVP (8 patients), P-Gemox (79 patients), CHOP or CHOP-like (60 patients).

Significant differences in chemotherapy regimens of patients was based on RDW ( $P=0.011)$; however, we found no significant associations between RDW and treatment response, chemotherapy cycles, and treatment modalities (Table 2).

\section{Survival and prognostic factors}

Of the 191 patients studied, 54 patients died after a median follow-up of 30 months (range $=2-97$ ). The 3 -year OS and PFS in the whole group were $75.4 \%$ and $72.8 \%$, respectively. The results of RDW distinguished two groups with significantly different survival outcomes (Figure 2). Patients with RDW $\leq 46.2 \mathrm{fL}$ had significantly better PFS than those with RDW>46.2 fL (3-year PFS, 80.4\% vs. $63.1 \% ; P=0.01$, Figure 2A) and OS (3-year OS, 83.2\% vs. $65.5 \% ; P=0.004$, Figure $2 \mathrm{~B}$ ). Due to the existence of significant differences in chemotherapy regimens of patients based on RDW, we conducted subgroup analysis. We discovered that RDW is a significant predictor of overall survival for patients treated with P-Gemox, LVD, or VDLP regimen, but not $\mathrm{CHOP}$ or $\mathrm{CHOP}$-like regimen ( $\mathrm{P}=0.032, \mathrm{P}=0.201$, respectively, Figure 3$)$. Nevertheless, patients harboring RDW $>46.2 \mathrm{fL}$ tended to have worse OS than those with RDW $\leq 46.2 \mathrm{fL}$ (3-year OS, 62.8\% vs. $83.3 \%$; mean OS, 51.9 months vs. 67.2 months). Thus, we still consider RDW a weak predictive index for patients with CHOP or CHOP-like regimen.

The results of univariate and multivariate analysis are displayed in Table 3. In the multivariate analysis for PFS, RDW >46.2 fL, HB>126 g/L, and local invasiveness were found to be significant independent predictors of PFS $(P=0.03, \mathrm{HR}=1.895 ; P=0.036, \mathrm{HR}=0.555 ; P=0.023$, $\mathrm{HR}=2.204$, respectively). Furthermore, $\mathrm{RDW}>46.2$ $\mathrm{fL}, \mathrm{HB}>126 \mathrm{~g} / \mathrm{L}$, and local invasiveness remained significantly predictive of $\mathrm{OS}(P=0.021, \mathrm{HR}=2.04$; $P=0.016, \mathrm{HR}=0.49 ; P=0.03, \mathrm{HR}=2.244$, respectively) in the multivariate analysis for OS. 
Table 1: Patient baseline characteristics by RDW Level

\begin{tabular}{|c|c|c|c|c|}
\hline Characteristics & Total, $\mathbf{n}=191(\%)$ & $\begin{array}{c}\mathrm{RDW} \leq 46.2 \mathrm{fL} \\
\mathrm{n}=107(\%)\end{array}$ & $\begin{array}{c}\mathrm{RDW}>46.2 \mathrm{fL} \\
\mathrm{n}=84(\%)\end{array}$ & $\mathbf{P}$ \\
\hline Age(median [range], yr) & $44(15,86)$ & & & 0.078 \\
\hline$\leq 60$ & $159(83.2)$ & $94(87.9)$ & $65(77.4)$ & \\
\hline$>60$ & $32(16.8)$ & $13(12.1)$ & $19(22.6)$ & \\
\hline Gender (male) & $134(70.2)$ & $75(70.1)$ & $59(70.2)$ & 1.00 \\
\hline ECOG PS & & & & $<0.001$ \\
\hline $0 \sim 1$ & $157(82.2)$ & $99(92.5)$ & $58(69.0)$ & \\
\hline$\geq 2$ & $34(17.8)$ & $8(7.5)$ & $26(31.0)$ & \\
\hline B symptoms (yes) & $88(46.1)$ & $50(46.7)$ & $38(45.2)$ & 0.884 \\
\hline $\mathrm{LDH}>240 \mathrm{U} / \mathrm{L}$ & $49(25.7)$ & $29(27.1)$ & $20(23.8)$ & 0.621 \\
\hline Extranodal sites $>2$ & $80(41.9)$ & $44(41.4)$ & $36(42.9)$ & 0.883 \\
\hline \multirow[t]{2}{*}{ Ki67 $>50 \%$} & Available (161) & Available (91) & Available (70) & 0.266 \\
\hline & $91(56.6)$ & $55(60.4)$ & $36(51.4)$ & \\
\hline $\mathrm{HB}(\leq 126 \mathrm{~g} / \mathrm{L})$ & $84(43.9 \%)$ & $45(42.1)$ & $39(46.4)$ & 0.560 \\
\hline Ann Arbor stage & & & & 1.00 \\
\hline $\mathrm{I} / \mathrm{II}$ & $184(96.3)$ & $103(96.3)$ & $81(96.3)$ & \\
\hline III/IV & $7(3.7)$ & $4(3.7)$ & $3(3.6)$ & \\
\hline Local invasiveness & $89(46.6)$ & $50(46.7)$ & $39(46.4)$ & 1.00 \\
\hline $\begin{array}{l}\text { Regional } \\
\text { lymphadenopathies }\end{array}$ & $80(41.9)$ & $52(48.6)$ & $28(33.3)$ & 0.039 \\
\hline \multicolumn{5}{|l|}{ Comorbidities } \\
\hline Smoking & $51(26.7)$ & $32(29.9)$ & $19(22.6)$ & 0.323 \\
\hline Drinking & $28(14.7)$ & $20(18.7)$ & $8(9.50)$ & 0.099 \\
\hline Hepatitis B & $12(6.30)$ & $7(6.50)$ & $5(6.00)$ & 1.00 \\
\hline Underlying diseases & $28(14.7)$ & $16(15.0)$ & $12(14.3)$ & 1.00 \\
\hline IPI score & & & & 0.009 \\
\hline $0-1$ & $139(72.8)$ & $86(80.4)$ & $53(63.1)$ & \\
\hline $2-5$ & $52(27.2)$ & 21(19.6) & $31(36.9)$ & \\
\hline PINK score & & & & 0.068 \\
\hline 0 & $153(80.1)$ & $91(85.0)$ & $62(73.8)$ & \\
\hline $1-3$ & $38(19.9)$ & $16(15.0)$ & $22(26.2)$ & \\
\hline KPI score & & & & 0.219 \\
\hline $0-1$ & $127(66.5)$ & $67(62.6)$ & $60(71.4)$ & \\
\hline $2-4$ & $64(33.5)$ & $40(37.4)$ & $24(28.6)$ & \\
\hline
\end{tabular}

NOTE. Regional lymphadenopathies defined as involvement of lymph nodes corresponding to N1-N3 of the primary lesion at the TNM staging system; local invasiveness defined as T3 or T4; Underlying diseases consisted of hypertension, hyperglycemia, hyperlipidemia and chronic bronchitis; Hepatitis B defined as serum HBsAg is positive. Characteristics with $\mathrm{P}$ values $<0.05$ are marked in bold. Abbreviations: ECOG PS, Eastern Cooperative Oncology Group performance status; HB, Hemoglobin; LDH, lactate dehydrogenase; EBER, EBV-encoded RNA; IPI, international prognostic index; KPI, Korean Prognostic Index; PINK, prognostic index of natural killer lymphoma. 


\section{Combining RDW to IPI, KPI, and PINK score improves survival prediction and risk stratification}

We conducted a model for combining RDW to the IPI, KPI, and PINK. Briefly, patients with an elevated RDW level (RDW $>46.2 \mathrm{fL}$ ) were allocated a score of 1 , while patients without an elevated RDW level received a score of 0 . We then added the RDW score to the IPI, KPI, and PINK scores to generate this new prognostic model. We performed C-index analysis to evaluate the discriminatory impact of RDW on OS. IPI and PINK scores were found to be significant with $\mathrm{C}$-index analysis in OS (0.607 and 0.537, respectively) (Figure 4A, 4C; Table 4). No significance was found between KPI and OS with a C-index of 0.588 (Figure 4B; Table 4). After combining RDW with the IPI, KPI, and PINK scores, the new prognostic model showed significant association with OS, and survival prediction and risk stratification were improved as indicated by $\mathrm{C}$-index $(0.640,0.639$, and 0.603 , respectively) (Figure 4D, 4F, 4E; Table 4).

Furthermore, subgroup analysis was conducted to further investigate the effect of RDW to IPI, KPI, and PINK score. The RDW failed to significant stratify patients with IPI scores $0-1(P>0.05$, data and figure not shown). However, the RDW distinguish patients with good outcomes from those with inferior outcomes among patients with IPI scores $\geq 2$ (Figure 5). For the KPI and PINK model, the RDW can stratify patients into a highrisk group or low-risk group (Figure 6; Figure 7).

Conversely, the IPI, KPI, and PINK prognostic models failed to show significant prognostic value among patients with $\mathrm{RDW}<46.2 \mathrm{fL}(P=0.876, P=0.683, P=0.596$, respectively, Figure 8 ).

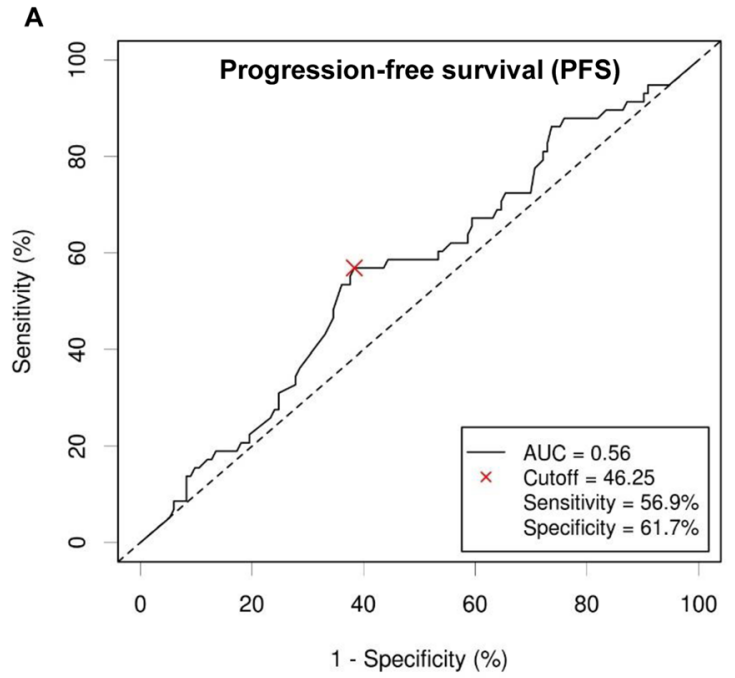

\section{DISCUSSION}

This retrospective study included 191 patients newly diagnosed with ENKTL to clarify the prognostic value of baseline RDW in pretreatment patients with ENKTL. Our results demonstrate that RDW is an independent prognostic factor for OS and PFS in patients with ENKTL given radiotherapy-based treatment. Moreover, combining RDW to IPI, KPI, and PINK scores can improve survival prediction and risk stratification. To our knowledge, this is the first report on the prognostic value of RDW in patients with ENKTL.

The RDW was used for the differential diagnosis of anemia for decades. However, in the recent years, RDW has gained notable renaissance. A variety of studies have found RDW to be a simple, robust, and convenient parameter associated with various human disorders such as cardiovascular disease [9], acute pulmonary embolism [10], diabetes [11], kidney disease [12, 13], liver disease [14], and chronic obstructive pulmonary disease (COPD) [15], but even more importantly, with overall mortality in the general population [3, 16, 17]. Recently, studies have demonstrated that RDW is prognostic factor for a variety of cancers [4]. Accumulating evidence has shown that RDW has important clinical significance. However, is RDW a risk factor or a simple epiphenomenon of an underlying biological or metabolic imbalance? Lippi et al. demonstrated that an elevated RDW involved both impaired erythropoiesis and abnormal erythrocyte metabolism and survival that mirrored a variety of abnormalities, such as shortening of telomeres length, oxidative stress, inflammation, erythrocyte fragmentation, poor nutritional status, hypertension, dyslipidemia and abnormality of erythropoietin function [3].

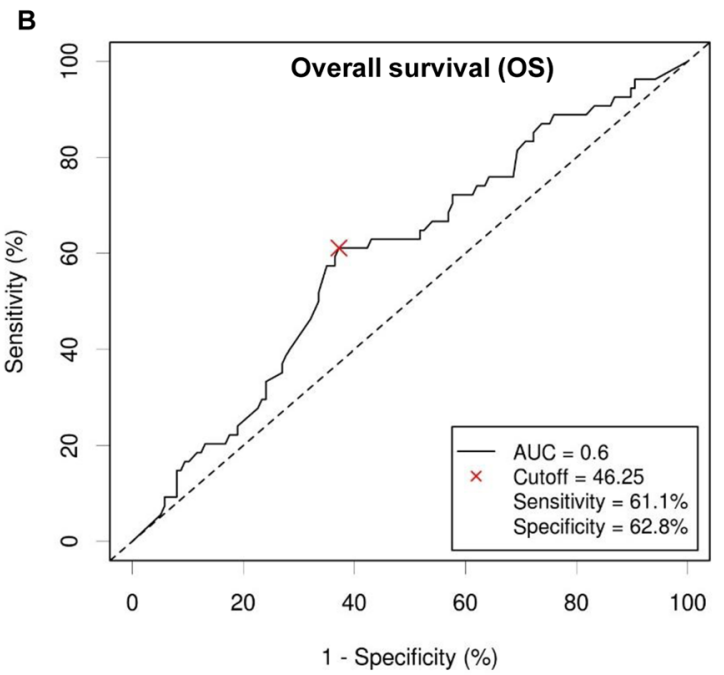

Figure 1: Receiver operating curve (ROC) from Cutoff Finder for determining the optimal cut-off value in predicting progression-free survival (PFS) (A) and overall survival (OS) (B) for red blood cell distribution width (RDW). 
Table 2: Treatment modalities and response in Patients with Extranodal Natural Killer (NK)/T-Cell Lymphoma

\begin{tabular}{|c|c|c|c|}
\hline Treatment & $\begin{array}{c}\text { RDW } \leq 46.2 \mathrm{fL}, \\
\text { n }(\%)\end{array}$ & $\begin{array}{c}\text { RDW }>46.2 \mathrm{fL} \\
\text { n }(\%)\end{array}$ & $\mathbf{P}$ \\
\hline Chemotherapy regimens & $86(100.0)$ & $65(100.0)$ & 0.011 \\
\hline CHOP or CHOP-like & $36(41.9)$ & $43(66.2)$ & \\
\hline P-Gemox & $46(53.5)$ & $14(21.5)$ & \\
\hline LVD/VDLP & $4(4.6)$ & $8(12.3)$ & \\
\hline Treatment outcome & $107(100.0)$ & $84(100.0)$ & 0.211 \\
\hline $\mathrm{CR}+\mathrm{PR}$ & $100(93.5)$ & $74(88.1)$ & \\
\hline $\mathrm{SD}+\mathrm{PD}$ & $7(6.5)$ & $10(11.9)$ & \\
\hline Chemotherapy cycles & $86(100.0)$ & $65(100.0)$ & 0.191 \\
\hline$<4$ & $38(44.2)$ & $26(55.4)$ & \\
\hline$\geq 4$ & $48(55.8)$ & $29(44.6)$ & \\
\hline Treatment modalities & $107(100.0)$ & $84(100.0)$ & 0.679 \\
\hline RT & $21(19.6)$ & $19(22.6)$ & \\
\hline CT followed by RT & $34(31.8)$ & $21(25.0)$ & \\
\hline Concurrent CRT & $11(10.3)$ & $11(13.1)$ & \\
\hline $\begin{array}{l}\text { Concurrent CRT followed } \\
\text { by CT }\end{array}$ & $8(7.5)$ & $6(7.1)$ & \\
\hline CT sandwiched RT & $27(25.2)$ & $18(21.4)$ & \\
\hline RT followed by CT & $6(5.6)$ & $9(10.7)$ & \\
\hline
\end{tabular}

Abbreviations: CHOP, cyclophosphamide + doxorubicin + vincristine + prednisone; P-Gemox, pegaspargase + gemcitabine + oxaliplatin; LVD, l-asparaginase + vincristine + prednisone; VDLP, etoposide + cisplatin + 1-asparaginase + dexamethasone; CR, complete response; PR, partial response; SD, stable disease; PD, progressive disease; CT, chemotherapy; RT, radiotherapy; CRT, Chemoradiotherapy.

Although the IPI, KPI and PINK are important prognostic models in patients with ENKTL with specific treatments, they have not been previously evaluated in ENKTL with radiotherapy-based treatment. In this study, they show significant association with OS and PFS in univariate analysis, whereas they also have some disadvantages. A shared disadvantage is that more than $70 \%$ of the cases were classified into the low-risk group, which is consistent with previous studies [18, 19]. The RDW can effectively divide the low-risk patients into two groups, yet it failed to significantly stratify patients with IPI scores $0-1$. Additional analysis found that the IPI, KPI and PINK were unable to clearly categorize high- and intermediate-risk groups. The high-risk group determined by IPI, KPI and PINK were further classified into two groups with significantly different survival outcomes with RDW. In order to investigate the impact of IPI, KPI, and PINK by RDW, these models were utilized to evaluate the RDW low-risk patients. All three models failed to significantly show significant prognostic value in patients with RDW $\leq 46.2 \mathrm{fL}$. Finally, we constructed a new model by combining RDW with the IPI, KPI, and PINK scores. The new model showed more powerful prognostic value than the corresponding original models. Thus, we have determined RDW as a novel and powerful prognostic factor, which may be more useful than previous prognostic models. This study merits further prospective and multicenter studies to validate the prognostic value of RDW in ENKTL.

Inflammatory conditions are present before or after an oncogenic change occurs [20]. Cancer-related inflammation is regarded as a hallmark characteristic of cancer development and progression [21]. Inflammatory cells release various signaling molecules in the tumor microenvironment, which serve as effectors of tumor cell proliferation, survival and migration [22]. Meanwhile, inflammation associated markers are frequently investigated to develop cost-effective predictors for patients with cancer [23-26]. Lippi et al. found that RDW is robustly associated with two most widely used plasma inflammatory indices: circulating highsensitivity C-reactive protein (hsCRP) and erythrocyte 
sedimentation rate (ESR) [27]. Additionally, in the healthy population and in patients with DLBCL, RDW was correlated with CRP $[8,28]$. RDW is also associated with nutritional indicators, including albumin, iron, folate, and vitamin B12 in patients with cancer [8, 29]. Though the mechanism of RDW association with cancer

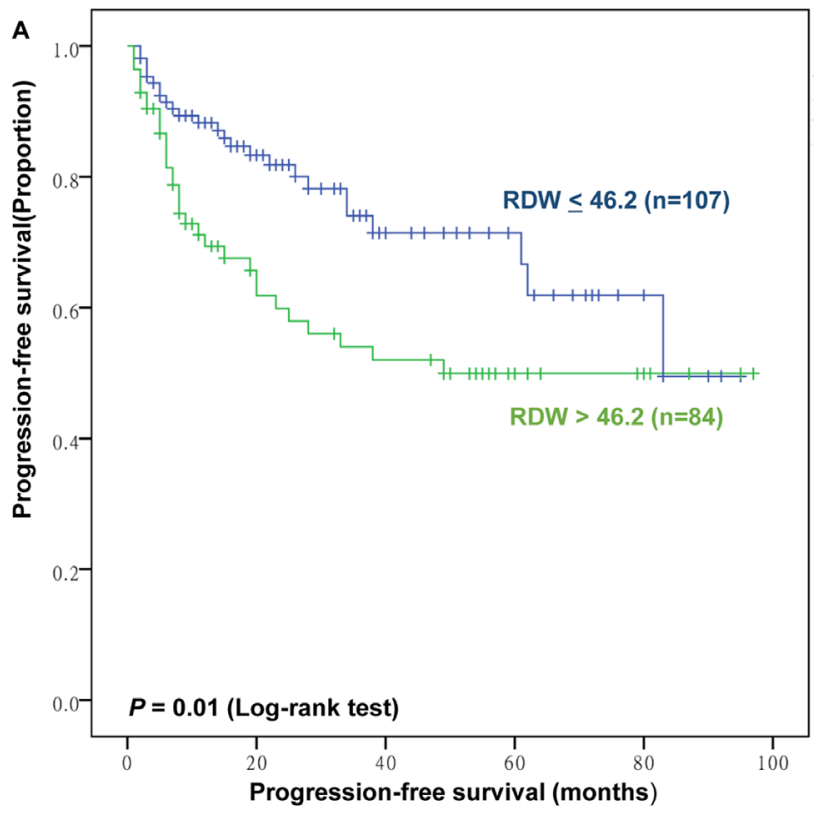

patient survival is unclear, a possible explanation is that high levels of RDW mirror underlying inflammatory and malnutrition conditions which weaken erythrocyte maturation and causes inadequate production of the hormone erythropoietin, malnutrition, or oxidative damage. As such, the decreased survival seen in patients

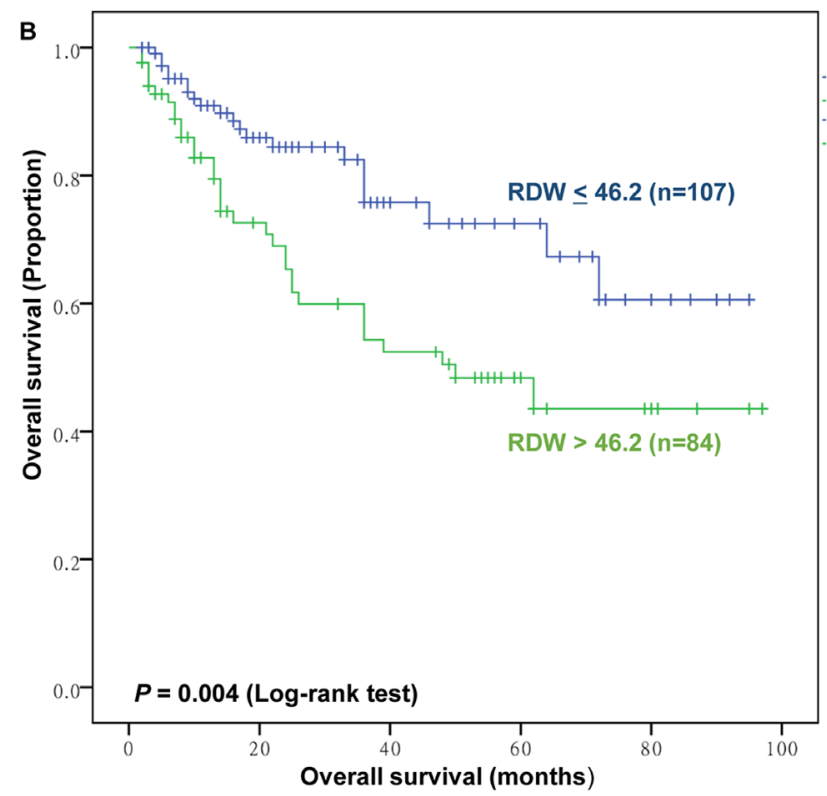

Figure 2: Prognostic value of red blood cell distribution width (RDW) for progression-free survival (PFS) and overall survival (OS). (A) PFS of patients according to RDW $<46.2 \mathrm{fL}$ versus $\mathrm{RDW}>46.2 \mathrm{fL}$. (B) $\mathrm{OS}$ of patients according to RDW $<46.2 \mathrm{fL}$ versus $R D W>46.2 \mathrm{fL}$.
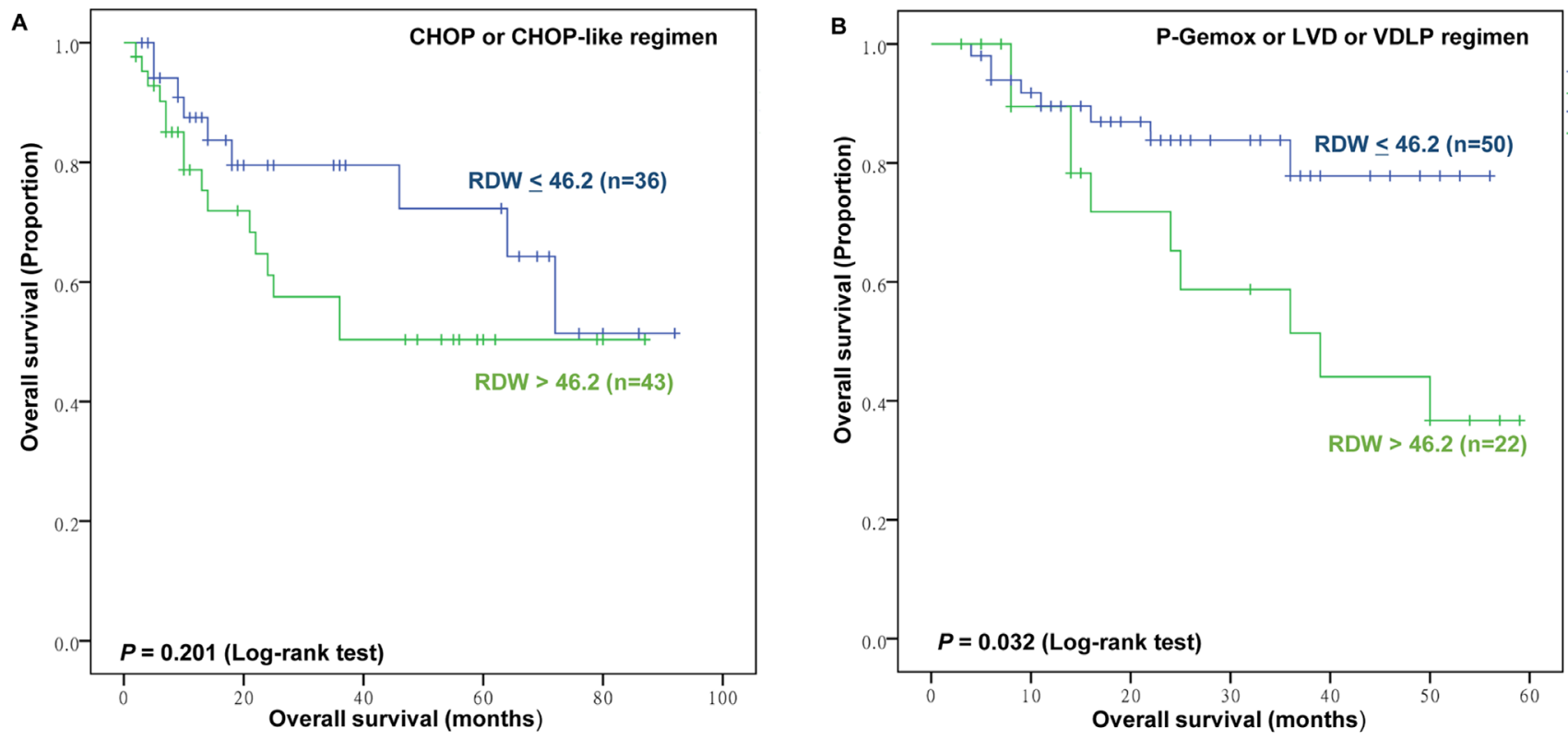

Figure 3: Subgroup analysis for prognostic value of red blood cell distribution width (RDW) for overall survival (OS). Kaplan-Meier plots for patients who received CHOP or CHOP-like (A) and non-CHOP or CHOP-like (P-Gemox or LVD or VDLP) (B) regimen according to $\mathrm{RDW}<46.2 \mathrm{fL}$ versus $\mathrm{RDW}>46.2 \mathrm{fL}$. 
Table 3: Univariate and Multivariate Analysis of Prognostic Factors for PFS and OS in Patients with ENKL

\begin{tabular}{|c|c|c|c|c|c|c|}
\hline \multirow{3}{*}{ Factors } & \multicolumn{3}{|c|}{ PFS } & \multicolumn{3}{|c|}{ OS } \\
\hline & \multirow{2}{*}{$\begin{array}{c}\begin{array}{c}\text { Univariate } \\
\text { analysis }\end{array} \\
P\end{array}$} & \multicolumn{2}{|c|}{ Multivariate analysis } & \multirow{2}{*}{$\begin{array}{c}\begin{array}{c}\text { Univariate } \\
\text { analysis }\end{array} \\
P\end{array}$} & \multicolumn{2}{|c|}{ Multivariate analysis } \\
\hline & & HR(95\%CI) & $P$ & & HR(95\%CI) & $P$ \\
\hline Age $>60$ years & 0.299 & & & 0.145 & & \\
\hline Gender, male & 0.608 & & & 0.763 & & \\
\hline ECOG PS >2 & $<0.001$ & & & $<0.001$ & & \\
\hline B symptoms, yes & 0.877 & & & 0.92 & & \\
\hline $\mathrm{LDH}>240 \mathrm{U} / \mathrm{L}$ & 0.03 & & & 0.026 & & \\
\hline Extranodal sites $>2$ & 0.001 & & & 0.001 & & \\
\hline Ki67 $>50 \%$ & 0.16 & & & 0.154 & & \\
\hline $\begin{array}{l}\text { Ann Arbor stage, } \\
\text { III/IV }\end{array}$ & 0.071 & & & 0.122 & & \\
\hline Local invasiveness & 0.004 & $2.204(1.117-4.349)$ & 0.023 & 0.009 & $2.244(1.08-4.663)$ & 0.03 \\
\hline $\begin{array}{l}\text { Regional } \\
\text { lymphadenopathies }\end{array}$ & 0.078 & & & 0.117 & & \\
\hline Smoking & 0.063 & & & 0.15 & & \\
\hline Drinking & 0.232 & & & 0.876 & & \\
\hline Hepatitis B & 0.511 & & & 0.605 & & \\
\hline $\mathrm{HB}>126 \mathrm{~g} / \mathrm{L}$ & 0.001 & $0.555(0.320-0.963)$ & 0.036 & $<0.001$ & $0.490(0.275-0.874)$ & 0.016 \\
\hline Underlying diseases & 0.007 & & & 0.002 & & \\
\hline IPI score >2 & $<0.001$ & & & $<0.001$ & & \\
\hline PINK score >1 & 0.042 & & & 0.019 & & \\
\hline KPI score >2 & 0.007 & & & 0.016 & & \\
\hline RDW > 46.2 fL & 0.01 & $1.895(1.063-3.380)$ & 0.030 & 0.004 & 2.04(1.115-3.746) & 0.021 \\
\hline
\end{tabular}

Abbreviations: ECOG PS, Eastern Cooperative Oncology Group performance status; HB, Hemoglobin; LDH, lactate dehydrogenase; EBER, EBV-encoded RNA; IPI, international prognostic index; KPI, Korean Prognostic Index; PINK, prognostic index of natural killer lymphoma; PFS, progression-free survival; OS, overall survival; Note: When the $P$ value lower than 0.05 , the corresponding factor was added into the multivariate analysis, and only the significant factors were listed for the results of multivariate analysis.

with elevated RDW may be a symptom of the underlying inflammation and malnutrition. Additional research is needed to explain the chemical and molecular mechanism relating RDW and cancer patient mortality.

According to National Comprehensive Cancer Network (NCCN) guidelines for Non-Hodgkin's lymphoma version 2.2015, the risk factors for ENKTL include age $>60$ years, ECOG PS score $\geq 2$, B symptoms, increased LDH, regional lymphadenopathies, local tumor invasion, histological expression of Ki-67, Epstein-Barr virus $(\mathrm{EBV}) \mathrm{DNA}>6.1 \mathrm{E}+7 / \mathrm{ml}$, and IPI score $>2$. With the exception of EBV DNA, due to insufficient data, the rest of the risk factors were evaluated in our study. While half of these risk factors show significant association with survival in the univariate analysis, four factors (age $>60$ years, B symptoms, regional lymphadenopathies and $\mathrm{Ki}-67>50 \%$ ) were not significantly prognostic for OS and PFS. Though age $>60$ years as a risk factor is investigated in many studies, some studies found that it was not predictive of survival [30-32]. Several previous studies also did not find significant associations between B symptoms and survival outcomes [33-35]. B symptoms was constructed in the pre-asparaginase era, and might lose its value in the era of asparaginase therapy. Regional lymphadenopathies was recognized as an independent risk indicator for worse OS in KPI [30]. Nevertheless, in our study, regional lymphadenopathies was not a significant predictor for OS, which is consistent with results presented 
C-index for OS

IPI

IPI+RDW

0.640

KPI

0.588

KPI+RDW

0.639

PINK

PINK+RDW

0.603

by Kim et al. [36]. This difference might be related to better local control in these two studies, because regional lymph nodes might be included in the radiation field. The relationship between Ki-67 expression and outcome with lymphoma showed strong heterogeneity among various studies [37]. In Au et al.'s study overall survival did not differ significantly between patients with and without Ki$67>50 \%$ in univariate analysis [19]. We also found that AA III/IV shows no association with OS and PFS of patients with ENKTL, which is inconsistent with the results of other studies $[30,36]$. This may result from the limited number of AA III/IV (7, 3.7\%) in our study and differences in primary treatment. However, a second study has reported results consistent with ours [19]. Notably, due to some common limitations such as retrospective research, sample size imbalance between Ann-Arbor staging I/II and III/IV, uniform treatment (radiotherapy-based treatment), clinical heterogeneity for ENKTL, and the fact that some risk factors show negative results in our study, further well designed prospective studies are necessary.

In conclusion, this study demonstrates a dependent association between high RDW and poorer clinical outcomes in ENKTL patients treated with radiotherapy-
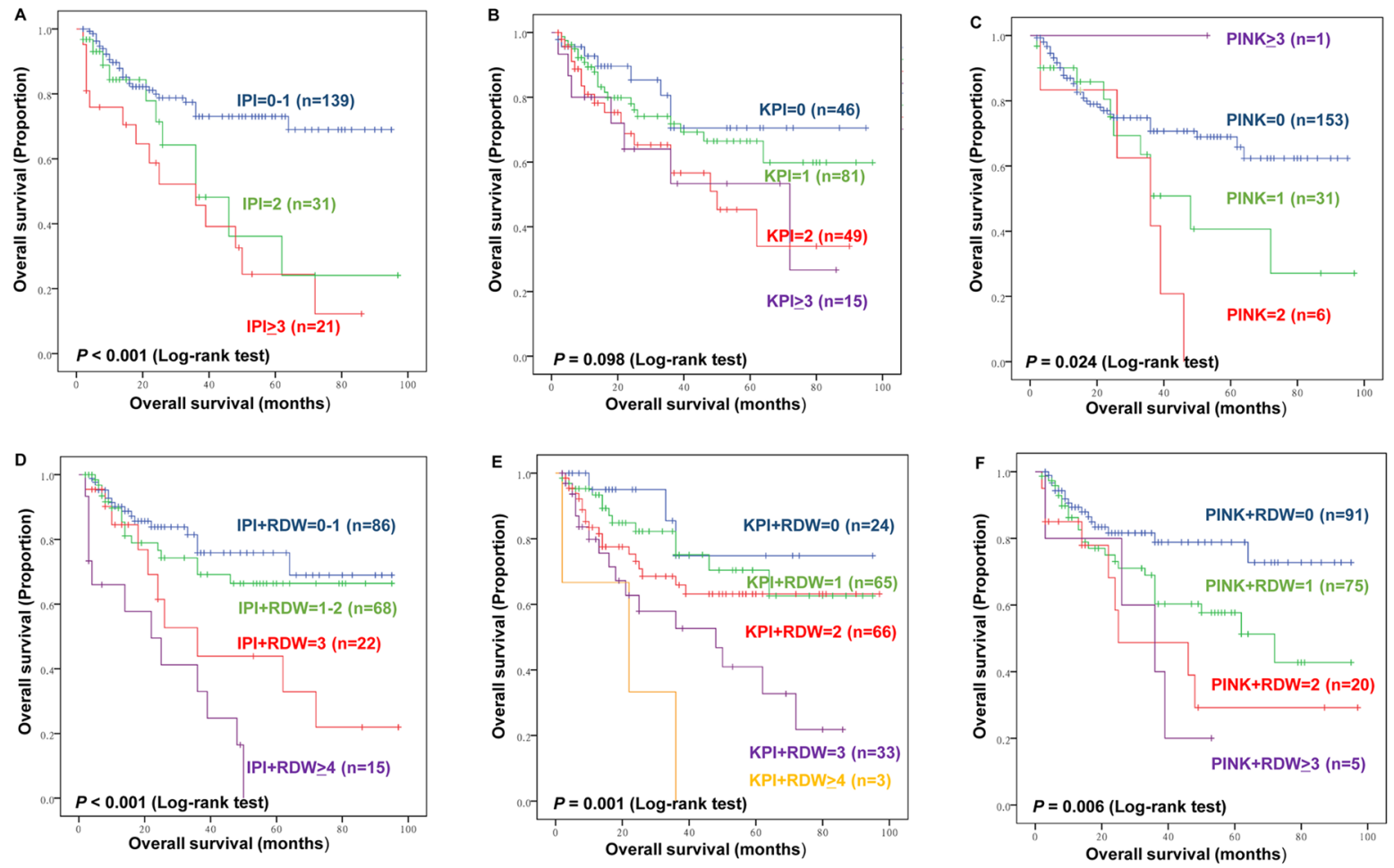

Figure 4: Kaplan-Meier plots of overall survival (OS) by International Prognostic Index (IPI) (A), Korean Prognostic Index (KPI) (B), Prognostic Index of Natural Killer lymphoma (PINK) (C), Kaplan-Meier plots of OS by the new model combining RDW and IPI (D), and KPI (E), and PINK (F). 

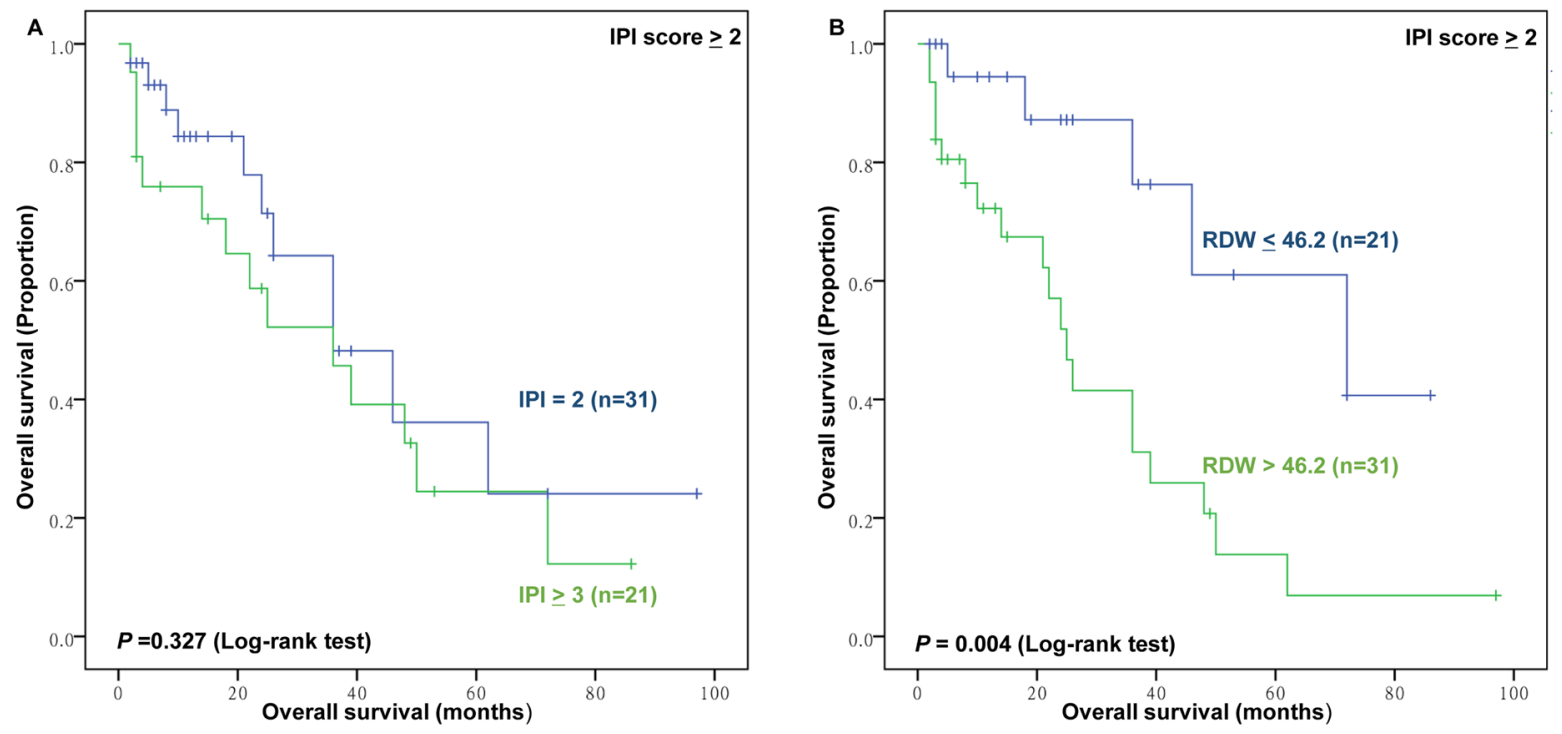

Figure 5: The prognostic value of high International Prognostic Index risk (IPI) and RDW in high IPI group. (A) Overall survival (OS) of patients with IPI score $\geq 2$ according to IPI; (B) OS of patients with high IPI (IPI, score $\geq 2$ ) according to RDW $\leq 46.2 \mathrm{fL}$ versus RDW $>46.2 \mathrm{fL}$.
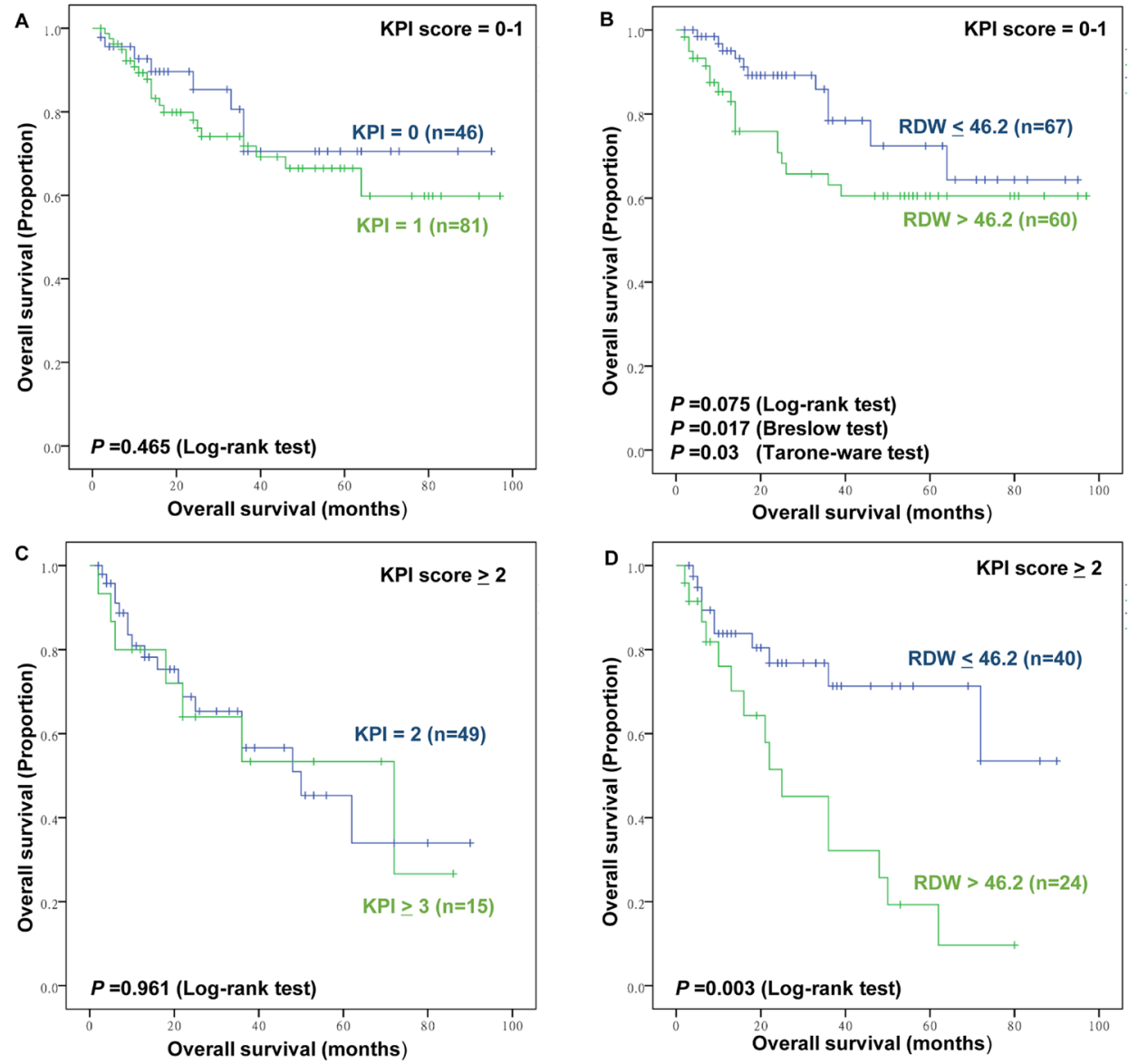

Figure 6: The prognostic value of Korean Prognostic Index (KPI) and RDW in low and high KPI group. (A) Overall survival (OS) of patients with low KPI (KPI, score 0-1) according to KPI (B) OS of patients with low KPI (KPI, score 0-1) according to RDW $\leq 46.2 \mathrm{fL}$ versus RDW $>46.2 \mathrm{fL}$. (C) OS of patients with high KPI (KPI, score $\geq 2$ ) according to KPI (D) OS of patients with high KPI (KPI, score $\geq 2$ ) according to $\mathrm{RDW} \leq 46.2 \mathrm{fL}$ versus $\mathrm{RDW}>46.2 \mathrm{fL}$. 

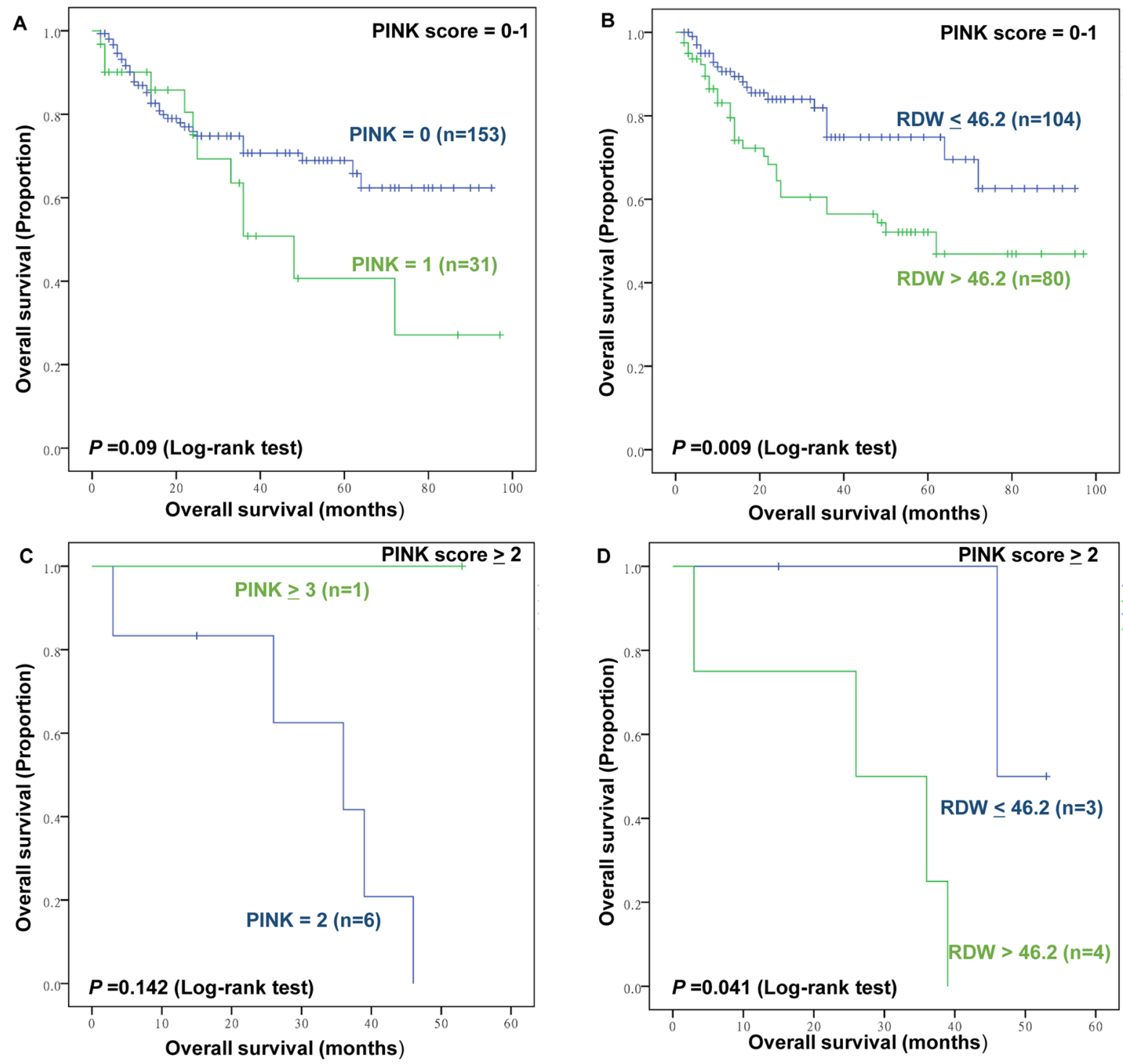

Figure 7: The prognostic value of Prognostic Index of Natural Killer lymphoma (PINK) and RDW in low and high PINK groups. (A) Overall survival (OS) of patients with low PINK (PINK, score 0-1) according to PINK; (B) OS of patients with low PINK (PINK, score 0-1) according to RDW $\leq 46.2 \mathrm{fL}$ versus RDW $>46.2 \mathrm{fL}$; (C) OS of patients with high PINK (PINK, score $\geq 2$ ) according to PINK (D) OS of patients with high PINK (KPI, score $\geq 2$ ) according to RDW $\leq 46.2 \mathrm{fL}$ versus RDW $>46.2 \mathrm{fL}$.
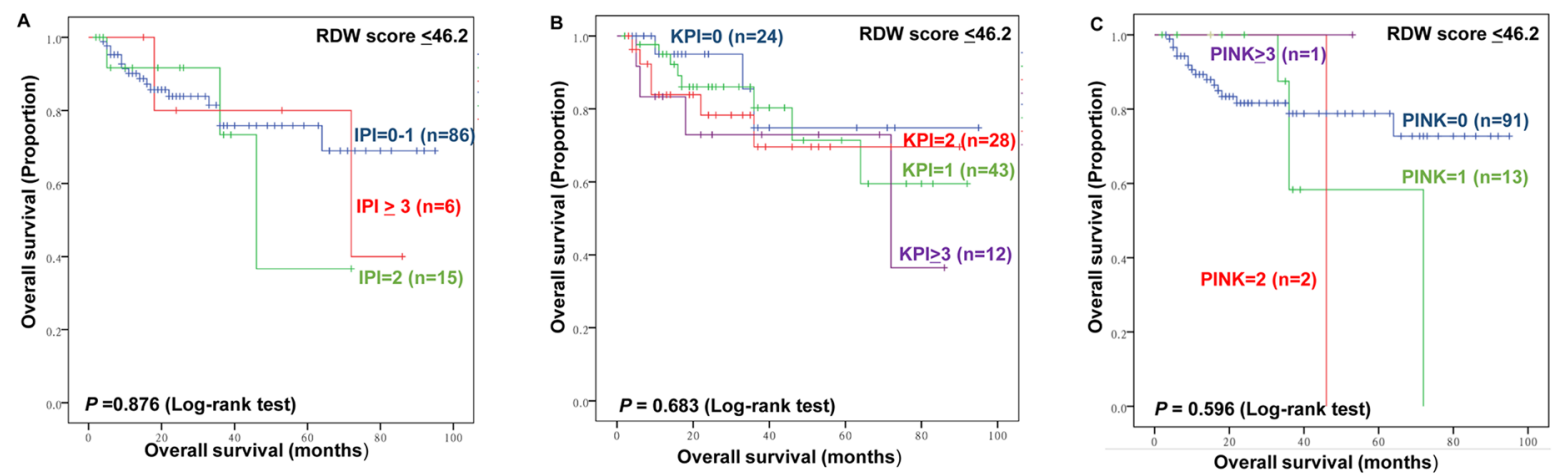

Figure 8: The impact of IPI (A), KPI (B) and PINK (C) by RDW $\leq 46.2 \mathrm{fL}$. 
based treatment. Further studies are warranted to confirm the prognostic value of RDW.

\section{MATERIALS AND METHODS}

\section{Patient selection and data collection}

We retrospectively enrolled 191 consecutive patients newly diagnosed with ENKTL in Sichuan Cancer Hospital between January 2008 and November 2016. All the patients included in this study were required to meet the following criteria: (1) pathologically and immunohistochemically confirmed diagnosis of ENKL, nasal type, by expert pathologists, according to World Health Organization (WHO) classifications [38], (2) available data regarding RDW at diagnosis, (3) sufficient clinical and follow-up data, (4) no acute infection or chronic active inflammatory disease, and (5) reception of radiotherapy-based treatment. This study was approved by the ethics committees of Sichuan cancer hospital. All patients consented to the use of their medical records for research purposes.

We collected the following pretreatment clinical and laboratory data: age, gender, physical examinations, Eastern Cooperative Oncology Group performance status (ECOG PS), B symptoms, serum lactate dehydrogenase (LDH), extranodal sites, Ki67, local invasiveness, regional lymphadenopathies, baseline RDW standard deviation (RDW-SD) levels, Ann Arbor (AA) stage, and comorbidities, including smoking, drinking, hemoglobin (HB) levels, hepatitis B (HBV), and other underlying diseases (including hypertension, hyperglycemia, hyperlipidemia and chronic bronchitis). International Prognostic Index (IPI) [39, 40], Korean Prognostic Index (KPI) [30], Prognostic Index of Natural Killer lymphoma (PINK) [36] for nasal NK/T-cell lymphoma were also used to perform survival analysis.

Regional lymphadenopathy was defined as the involvement of lymph nodes corresponding to N1-N3 of the primary lesion at the (Tumor, Node, Metastasis) TNM staging system. Local invasiveness was defined as T3 or T4 for upper aerodigestive tract NK/T-cell lymphoma (UNKTL) according to the 2002 TNM classification of the American Joint Committee on Cancer [30]. RBC analysis and RDW calculation was conducted on Mindray BC5800.

RDW was defined as the standard deviation (SD) of RBC volumes. Computed tomography (CT) or magnetic resonance (MR) of nasopharynx, neck, chest, and whole abdomen or positron emission tomography/CT (PET/CT) were utilized to evaluate patients' disease.

\section{Treatment}

The first-line chemotherapy regimens included non-anthracycline-based and anthracycline-containing regimens. The non-anthracycline-based regimens consisted of P-Gemox (pegaspargase, gemcitabine, and oxaliplatin), LVD (l-asparaginase, vincristine, and prednisone), and VDLP (etoposide, cisplatin, 1-asparaginase, dexamethasone). The anthracycline-containing regimen mainly included CHOP (cyclophosphamide, doxorubicin, vincristine, and prednisolone). A total dose of 50-56 Gy was given to involved-field radiotherapy, with daily fractions of 2 Gy based on conventional fractionation (five fractions per week).

Patients received one of the following initial treatment modalities: (1) radiotherapy alone, (2) chemotherapy followed by radiotherapy, (3) concurrent chemoradiotherapy, (4) concurrent chemoradiotherapy followed by chemotherapy, (5) chemotherapy before and after radiotherapy, and (6) radiotherapy followed by chemotherapy. The treatment response was evaluated according to the standard response criteria for nonHodgkin's lymphoma [41].

\section{Statistical analysis}

All statistical analyses were conducted with IBM SPSS 20.0 (SPSS Inc., Chicago, IL), R software version 3.2.3, and Cutoff Finder. A web-based R software (Cutoff Finder, http://molpath.charite.de/cutoff) was used to determine the optimal cut-off value for RDW and HB [42]. Overall survival (OS) was defined as the period from clear diagnosis to death, lost follow-up or deadline. Progression free survival (PFS) was defined as the period from clear diagnosis of the tumor to first tumor progression, death, lost follow-up or deadline. Fisher's exact test was used to identify associations between categorical variables. The Kaplan-Meier method was used to investigate OS and PFS, and the log-rank test was used to evaluate the difference. When the $P$ value of log-rank was close to 0.05 , breslow test and tarone-ware test were utilized. When the $P$ value was lower than 0.05 , the corresponding factor was added into the multivariate analysis. Multivariate analysis was conducted by Cox proportional hazard model. Discrimination for survival data was investigated utilizing the $\mathrm{C}$ statistic with concordance index (C-index) $[43,44]$. The C-index can evaluate the model's ability to classify individual patients into risk groups with different prognoses by estimating the probability of concordance between predicted and observed outcomes. C-index was calculated using Hmisc $\mathrm{R}$ package in $\mathrm{R}$ software version 3.2.3 [45]. A two tailed $\mathrm{P}$ value $<0.05$ was considered statistically significant.

\section{Abbreviations}

RDW: red blood cell distribution width, HR: hazard ratio, CI: confidence interval, OS: overall survival, PFS: progression-free survival, IPI: International prognostic index, KPI: Korean Prognostic Index, PINK: prognostic index of natural killer lymphoma, ECOG PS: Eastern 
Cooperative Oncology Group performance status, LDH: lactate dehydrogenase, CHOP: cyclophosphamide + doxorubicin + vincristine + prednisone, P-Gemox: pegaspargase + gemcitabine + oxaliplatin, LVD: l-asparaginase + vincristine + prednisone, VDLP: etoposide + cisplatin +1 -asparaginase + dexamethasone.

\section{ACKNOWLEDGMENTS AND FUNDING}

This study was supported by grants from Health and Family Planning Commission of Sichuan Province universal application project (17PJ516). We thank Michelle Wei from the Duke University for its linguistic assistance during the preparation of this manuscript and the reviewers for their intellectual support.

\section{CONFLICTS OF INTEREST}

The authors have no conflicts of interests to declare.

\section{REFERENCES}

1. Haverkos BM, Pan Z, Gru AA, Freud AG, Rabinovitch R, Xu-Welliver M, Otto B, Barrionuevo C, Baiocchi RA, Rochford R, Porcu P. Extranodal NK/T Cell Lymphoma, Nasal Type (ENKTL-NT): An Update on Epidemiology, Clinical Presentation, and Natural History in North American and European Cases. Curr Hematol Malig Rep. 2016; 11:514-27.

2. Li YX, Liu QF, Fang H, Qi SN, Wang H, Wang WH, Song YW, Lu J, Jin J, Wang SL, Liu YP, Lu N, Liu XF, Yu ZH. Variable clinical presentations of nasal and Waldeyer ring natural killer/T-cell lymphoma. Clin Cancer Res. 2009; 15:2905-12.

3. Salvagno GL, Sanchis-Gomar F, Picanza A, Lippi G. Red blood cell distribution width: A simple parameter with multiple clinical applications. Crit Rev Clin Lab Sci. 2015; 52:86-105.

4. Hu L, Li M, Ding Y, Pu L, Liu J, Xie J, Cabanero M, Li J, Xiang R, Xiong S. Prognostic value of RDW in cancers: a systematic review and meta-analysis. Oncotarget. 2017; 8:16027-16035. https://doi.org/10.18632/oncotarget.13784.

5. Huang DP, Ma RM, Xiang YQ. Utility of Red Cell Distribution Width as a Prognostic Factor in Young Breast Cancer Patients. Medicine (Baltimore). 2016; 95:e3430.

6. Sun P, Zhang F, Chen C, Bi X, Yang H, An X, Wang F, Jiang $\mathrm{W}$. The ratio of hemoglobin to red cell distribution width as a novel prognostic parameter in esophageal squamous cell carcinoma: a retrospective study from southern China. Oncotarget. 2016; 7:42650-60. https://doi.org/10.18632/ oncotarget.9516.

7. Koma Y, Onishi A, Matsuoka H, Oda N, Yokota N, Matsumoto Y, Koyama M, Okada N, Nakashima N, Masuya D, Yoshimatsu H, Suzuki Y. Increased red blood cell distribution width associates with cancer stage and prognosis in patients with lung cancer. PLoS One. 2013; 8:e80240.

8. Periša V, Zibar L, Sinčić-Petričević J, Knezović A, Periša I, Barbić J. Red blood cell distribution width as a simple negative prognostic factor in patients with diffuse large B-cell lymphoma: a retrospective study. Croat Med J. 2015; 56:334-43.

9. Lippi G, Filippozzi L, Montagnana M, Salvagno GL, Franchini M, Guidi GC, Targher G. Clinical usefulness of measuring red blood cell distribution width on admission in patients with acute coronary syndromes. Clin Chem Lab Med. 2009; 47:353-57.

10. Zorlu A, Bektasoglu G, Guven FM, Dogan OT, Gucuk E, Ege MR, Altay H, Cinar Z, Tandogan I, Yilmaz MB. Usefulness of admission red cell distribution width as a predictor of early mortality in patients with acute pulmonary embolism. Am J Cardiol. 2012; 109:128-34.

11. Veeranna V, Zalawadiya SK, Panaich SS, Ramesh K, Afonso L. The association of red cell distribution width with glycated hemoglobin among healthy adults without diabetes mellitus. Cardiology. 2012; 122:129-32.

12. Lippi G, Targher G, Montagnana M, Salvagno GL, Zoppini G, Guidi GC. Relationship between red blood cell distribution width and kidney function tests in a large cohort of unselected outpatients. Scand J Clin Lab Invest. 2008; 68:745-48.

13. Oh HJ, Park JT, Kim JK, Yoo DE, Kim SJ, Han SH, Kang SW, Choi KH, Yoo TH. Red blood cell distribution width is an independent predictor of mortality in acute kidney injury patients treated with continuous renal replacement therapy. Nephrol Dial Transplant. 2012; 27:589-94.

14. Beyazit Y, Kekilli M, Ibis M, Kurt M, Sayilir A, Onal IK, Purnak T, Oztas E, Tas A, Yesil Y, Arhan M. Can red cell distribution width help to discriminate benign from malignant biliary obstruction? A retrospective single center analysis. Hepatogastroenterology. 2012; 59:1469-73.

15. Braun E, Domany E, Kenig Y, Mazor Y, Makhoul BF, Azzam ZS. Elevated red cell distribution width predicts poor outcome in young patients with community acquired pneumonia. Crit Care. 2011; 15:R194.

16. Patel KV, Ferrucci L, Ershler WB, Longo DL, Guralnik JM. Red blood cell distribution width and the risk of death in middle-aged and older adults. Arch Intern Med. 2009; 169:515-23.

17. Perlstein TS, Weuve J, Pfeffer MA, Beckman JA. Red blood cell distribution width and mortality risk in a community-based prospective cohort. Arch Intern Med. 2009; 169:588-94.

18. Huang JJ, Zhu YJ, Xia Y, Zhao W, Lin TY, Jiang WQ, Huang HQ, Li ZM. A novel prognostic model for extranodal natural killer/T-cell lymphoma. Med Oncol. 2012; 29:2183-90. 
19. Au WY, Weisenburger DD, Intragumtornchai $\mathrm{T}$, Nakamura S, Kim WS, Sng I, Vose J, Armitage JO, Liang R, and International Peripheral T-Cell Lymphoma Project. Clinical differences between nasal and extranasal natural killer/Tcell lymphoma: a study of 136 cases from the International Peripheral T-Cell Lymphoma Project. Blood. 2009; 113:3931-37.

20. Mantovani A, Allavena P, Sica A, Balkwill F. Cancer-related inflammation. Nature. 2008; 454: 436-44.

21. Hanahan D, Weinberg RA. The hallmarks of cancer. Cell. 2000; 100:57-70.

22. Hanahan D, Weinberg RA. Hallmarks of cancer: the next generation. Cell. 2011; 144: 646-74.

23. Yang X, Huang Y, Feng JF, Liu JS. Prognostic significance of neutrophil-to- lymphocyte ratio in esophageal cancer: a meta-analysis. Onco Targets Ther. 2015; 8:789-94.

24. Pine JK, Fusai KG, Young R, Sharma D, Davidson BR, Menon KV, Rahman SH. Serum C-reactive protein concentration and the prognosis of ductal adenocarcinoma of the head of pancreas. Eur J Surg Oncol. 2009; 35:605-10.

25. Allin KH, Nordestgaard BG. Elevated C-reactive protein in the diagnosis, prognosis, and cause of cancer. Crit Rev Clin Lab Sci. 2011; 48:155-70.

26. Zhou X, Du Y, Huang Z, Xu J, Qiu T, Wang J, Wang T, Zhu W, Liu P. Prognostic value of PLR in various cancers: a meta-analysis. PLoS One. 2014; 9:e101119.

27. Lippi G, Targher G, Montagnana M, Salvagno GL, Zoppini G, Guidi GC. Relation between red blood cell distribution width and inflammatory biomarkers in a large cohort of unselected outpatients. Arch Pathol Lab Med. 2009; 133:628-32.

28. Vayá A, Sarnago A, Fuster O, Alis R, Romagnoli M. Influence of inflammatory and lipidic parameters on red blood cell distribution width in a healthy population. Clin Hemorheol Microcirc. 2015; 59:379-85.

29. Zhao T, Cui L, Li A. The significance of RDW in patients with hepatocellular carcinoma after radical resection. Cancer Biomark. 2016; 16:507-12.

30. Lee J, Suh C, Park YH, Ko YH, Bang SM, Lee JH, Lee DH, Huh J, Oh SY, Kwon HC, Kim HJ, Lee SI, Kim JH, et al. Extranodal natural killer T-cell lymphoma, nasal-type: a prognostic model from a retrospective multicenter study. $\mathrm{J}$ Clin Oncol. 2006; 24:612-18.

31. Li N, Zhang L, Song HL, Zhang J, Weng HW, Zou LQ. Prognostic impact of absolute lymphocyte count/absolute monocyte count ratio and prognostic score in patients with nasal-type, extranodal natural killer/T-cell lymphoma. Tumour Biol. 2017; 39:1010428317705503.

32. Jo JC, Yoon DH, Kim S, Lee BJ, Jang YJ, Park CS, Huh J, Lee SW, Ryu JS, Suh C. Clinical features and prognostic model for extranasal NK/T-cell lymphoma. Eur J Haematol. 2012; 89:103-10.

33. Wang L, Xia ZJ, Lu Y, Wang QX, Niu SQ, Huang HQ, Zhang YJ. A modified international prognostic index including pretreatment hemoglobin level for early stage extranodal natural killer/T cell lymphoma. Leuk Lymphoma. 2015; 56:3038-44.

34. Wang L, Xia ZJ, Huang HQ, Lu Y, Zhang YJ. Cyclophosphamide, doxorubicin, vincristine, and prednisone (CHOP) in the treatment of stage IE/IIE extranodal natural killer/T cell lymphoma, nasal type: 13-year follow-up in 135 patients. Int J Hematol. 2012; 96:617-23.

35. Wang L, Wang WD, Xia ZJ, Zhang YJ, Xiang J, Lu Y. Combination of gemcitabine, L-asparaginase, and oxaliplatin (GELOX) is superior to EPOCH or CHOP in the treatment of patients with stage IE/IIE extranodal natural killer/T cell lymphoma: a retrospective study in a cohort of 227 patients with long-term follow-up. Med Oncol. 2014; 31:860.

36. Kim SJ, Yoon DH, Jaccard A, Chng WJ, Lim ST, Hong H, Park Y, Chang KM, Maeda Y, Ishida F, Shin DY, Kim JS, Jeong SH, et al. A prognostic index for natural killer cell lymphoma after non-anthracycline-based treatment: a multicentre, retrospective analysis. Lancet Oncol. 2016; 17:389-400.

37. He X, Chen Z, Fu T, Jin X, Yu T, Liang Y, Zhao X, Huang L. Ki-67 is a valuable prognostic predictor of lymphoma but its utility varies in lymphoma subtypes: evidence from a systematic meta-analysis. BMC Cancer. 2014; 14:153.

38. Sabattini E, Bacci F, Sagramoso C, Pileri SA. WHO classification of tumours of haematopoietic and lymphoid tissues in 2008: an overview. International Agency for Research on Cancer. 2008.

39. Shipp M, Harrington D, Anderson J, Armitage JO, Bonadonna G, Brittinger G, Cabanillas F, Canellos G, Coiffier B, Connors J, and International Non-Hodgkin's Lymphoma Prognostic Factors Project. A predictive model for aggressive non-Hodgkin's lymphoma. N Engl J Med. 1993; 329:987-94.

40. Chim CS, Ma SY, Au WY, Choy C, Lie AK, Liang R, Yau CC, Kwong YL. Primary nasal natural killer cell lymphoma: long-term treatment outcome and relationship with the International Prognostic Index. Blood. 2004; 103:216-21.

41. Cheson BD, Horning SJ, Coiffier B, Shipp MA, Fisher RI, Connors JM, Lister TA, Vose J, Grillo-López A, Hagenbeek A, Cabanillas F, Klippensten D, Hiddemann W, et al, and NCI Sponsored International Working Group. Report of an international workshop to standardize response criteria for non-Hodgkin's lymphomas. J Clin Oncol. 1999; 17:1244.

42. Budczies J, Klauschen F, Sinn BV, Győrffy B, Schmitt WD, Darb-Esfahani S, Denkert C. Cutoff Finder: a comprehensive and straightforward Web application enabling rapid biomarker cutoff optimization. PLoS One. 2012; 7:e51862.

43. Harrell FE Jr, Lee KL, Mark DB. Multivariable prognostic models: issues in developing models, evaluating assumptions and adequacy, and measuring and reducing errors. Stat Med. 1996; 15:361-87. 
44. Keam B, Ha H, Kim TM, Jeon YK, Lee SH, Kim DW, Kim CW, Heo DS. Neutrophil to lymphocyte ratio improves prognostic prediction of International Prognostic Index for patients with diffuse large B-cell lymphoma treated with rituximab, cyclophosphamide, doxorubicin, vincristine and prednisone. Leuk Lymphoma. 2015; 56:2032-38.
45. Harrell FE Jr, Califf RM, Pryor DB, Lee KL, Rosati RA. Evaluating the yield of medical tests. JAMA. 1982; 247:2543-46. 